\title{
Some Statistical Parameters Related to the Nakagami-Rice Probability Distribution
}

\author{
William R. Burns \\ Contribution from Central Radio Propagation Laboratory, National Bureau of Standards, \\ Boulder, Colo.
}

(Received November 22, 1963)

\begin{abstract}
Formulas and tables are given for the mean and standard deviation of $R=20 \log _{10} r$ where the random variable $r$ has the Nakagami-Rice distribution. This distribution is of interest in connection with the short-term fading characteristics of some received radio fields. A particularly simple formula for the mean of $R$ is obtained in terms of the well-known exponential integral function $-E i(-x)$. Additional information concerning the median and interdecile range of $R$ is also given.
\end{abstract}

\section{Introduction}

In certain radio propagation problems the field strength at a receiver may be approximated by the vector sum of a constant vector and a Rayleigh-distributed vector [Norton, Vogler, Mansfield, and Short, 1955]. If it is assumed that the Rayleigh-distributed vector has an rms amplitude $k$ and that the constant vector has an rms amplitude of unity, then the probability distribution of the resultant $r$ of their sum as given by Nakagami [1940] and Rice [1944, 1945] may be put in the form

$$
P\left(r>r_{0}\right)=\frac{2}{k^{2}} \int_{r_{0}}^{\infty} r \exp \left[-\left(1+r^{2}\right) / k^{2}\right] I_{0}\left(2 r / k^{2}\right) d r
$$

where $I_{0}(x)$ is the modified Bessel function of first kind and order zero. If the Rayleigh distributed vector has an rms amplitude $k_{1}$, the constant vector has an rms amplitude $k_{2}$, and the resultant of their sum is $r_{1}$, then (1.1) gives the distribution of $r=r_{1} / k_{2}$ as a function of $k=k_{1} / k_{2}$.

The primary purpose of this paper is the determination of the mean $\bar{R}$ and standard deviation $\sigma_{R}$ of $R=20 \log _{10} r$. Additional information concerning the median $R(0.5)$ and interdecile range $R(0.1)-R(0.9)$ of $R$ is taken from the paper by Norton et al. [1955]. The phase $\phi$ of the vector sum of a Rayleigh-distributed vector and a constant vector is discussed by Norton, Shultz, and Yarbrough [1952].

\section{Calculation of $\bar{R}$ and $\sigma_{R}$}

We will determine $\sigma_{R}$ from the relation

$$
\sigma_{R}^{2}=\overline{R^{2}}-(\bar{R})^{2}
$$

where $\overline{R^{2}}$ is the mean of $R^{2}$. Making use of (1.1), we find that

and

$$
\bar{R}=\frac{2}{k^{2}} \int_{0}^{\infty}\left(20 \log _{10} r\right) r \exp \left[-\left(1+r^{2}\right) / k^{2}\right] I_{0}\left(2 r / k^{2}\right) d r
$$

$$
\overline{R^{2}}=\frac{2}{k^{2}} \int_{0}^{\infty}\left(20 \log _{10} r\right)^{2} r \exp \left[-\left(1+r^{2}\right) / k^{2}\right] I_{0}\left(2 r / k^{2}\right) d r
$$

In appendix 1 of this paper, these two integrals are evaluated and the three equations which follow are derived.

$$
\bar{R}=\left(10 \log _{10} e\right)\left[-E i\left(-1 / k^{2}\right)\right] .
$$


For $k \geq 1$, use

$$
\sigma_{R}^{2}=\left(10 \log _{10} e\right)^{2}\left\{\frac{\pi^{2}}{6}+2 \sum_{n=1}^{\infty} \frac{(-1)^{n+1}\left(1 / k^{2}\right)^{n+1}}{(n+1) !(n+1)}\left(\sum_{j=1}^{n} \frac{1}{j}\right)-\left[\sum_{n=1}^{\infty} \frac{(-1)^{n-1}\left(1 / k^{2}\right)^{n}}{n ! n}\right]^{2}\right\} .
$$

For $k<1$, use

$$
\begin{aligned}
\sigma_{R}^{2}=\left(10 \log _{10} e\right)^{2} & \left\{-2 E i\left(-1 / k^{2}\right) E^{*}\left(1 / k^{2}\right)+4 \int_{1}^{1 / k^{2}} \frac{-E i(-t)}{t} d t\right. \\
& \left.-2 \int_{1}^{1 / k^{2}} \frac{e^{t}[-E i(-t)]}{t} d t-\left[E i\left(-1 / k^{2}\right)\right]^{2}+4 E i\left(-1 / k^{2}\right)\left[\gamma+\ln \left(1 / k^{2}\right)\right]+C_{1}\right\} .
\end{aligned}
$$

In these equations,

$$
\begin{aligned}
-E i(-x) & =\int_{x}^{\infty} \frac{e^{-t}}{t} d t, \quad x>0 ; \\
E^{*}(x) & =-{\frac{\Gamma}{J_{-x}}}^{\infty} \frac{e^{-t}}{t} d t, \quad x>0,
\end{aligned}
$$

where

$$
{\frac{\Gamma^{\prime}}{J_{-x}}}^{\infty}=\lim _{\epsilon \rightarrow 0}\left(\int_{-x}^{-\epsilon}+\int_{\epsilon}^{\infty}\right)
$$

with $\epsilon>0$; and $C_{1}=1.099019$, a constant. The functions $-E i(-x)$ and $E^{*}(x)$ are well-known functions for which tables are available. See, for example, the book "Tables of Sine, Cosine, and Exponential Integrals," U.S. National Bureau of Standards [1940]. The integrals

$$
\int_{1}^{1 / k^{2}} \frac{E i(-t)}{t} d t
$$

and

$$
\int_{1}^{1 / k^{2}} \frac{e^{t}}{t}[-E i(-t)] d t
$$

\begin{tabular}{|c|c|c|c|c|}
\hline$K$ & $R(0.5)$ & $\bar{R}$ & $\bar{R}-R(0.5)$ & $\sigma_{R}$ \\
\hline $\begin{array}{l}-40 \\
-35 \\
-30 \\
-25 \\
-20\end{array}$ & $\begin{array}{r}0.000 \\
.001 \\
.002 \\
.007 \\
.022\end{array}$ & $\begin{array}{r}0.000 \\
.000 \\
.000 \\
.000 \\
.000\end{array}$ & $\begin{array}{r}-0.000 \\
-.001 \\
-.002 \\
-.007 \\
-.022\end{array}$ & $\begin{array}{r}0.061 \\
.109 \\
.194 \\
.346 \\
.616\end{array}$ \\
\hline $\begin{array}{l}-18 \\
-16 \\
-14 \\
-12 \\
-10\end{array}$ & $\begin{array}{l}.034 \\
.054 \\
.086 \\
.136 \\
.214\end{array}$ & $\begin{array}{l}.000 \\
.000 \\
.000 \\
.000 \\
.000\end{array}$ & $\begin{array}{l}-.034 \\
-.054 \\
-.086 \\
-.136 \\
-.214\end{array}$ & $\begin{array}{r}.776 \\
.980 \\
1.238 \\
1.569 \\
1.999\end{array}$ \\
\hline $\begin{array}{r}-8 \\
-6 \\
-4 \\
-2 \\
0\end{array}$ & $\begin{array}{r}.335 \\
.524 \\
.813 \\
1.249 \\
1.894\end{array}$ & $\begin{array}{l}.001 \\
.017 \\
.107 \\
.383 \\
.953\end{array}$ & $\begin{array}{l}-.334 \\
-.507 \\
-.706 \\
-.866 \\
-.941\end{array}$ & $\begin{array}{l}\text { 2. } 565 \\
\text { 3. } 279 \\
4.036 \\
\text { 4. } 667 \\
5.094\end{array}$ \\
\hline $\begin{array}{r}2 \\
4 \\
6 \\
8 \\
10\end{array}$ & $\begin{array}{l}2.808 \\
4.006 \\
5.448 \\
7.077 \\
8.835\end{array}$ & $\begin{array}{l}1.855 \\
3.064 \\
4.519 \\
6.155 \\
7.917\end{array}$ & $\begin{array}{l}-.953 \\
-.942 \\
-.929 \\
-.922 \\
-.918\end{array}$ & $\begin{array}{l}5.340 \\
5.465 \\
5.525 \\
5.551 \\
5.562\end{array}$ \\
\hline $\begin{array}{l}12 \\
14 \\
16 \\
18 \\
20 \\
\infty\end{array}$ & $\begin{array}{c}10.679 \\
12.580 \\
14.517 \\
16.477 \\
18.452 \\
\infty\end{array}$ & $\begin{array}{r}9.763 \\
11.664 \\
13.602 \\
15.562 \\
17.537 \\
\infty\end{array}$ & $\begin{array}{l}-.916 \\
-.916 \\
-.915 \\
-.915 \\
-.915 \\
-.915\end{array}$ & $\begin{array}{l}5.567 \\
5.569 \\
5.570 \\
5.570 \\
5.570 \\
5.570\end{array}$ \\
\hline
\end{tabular}

are evaluated in appendix 2 to six significant digits by numerical integration.

Letting $K=20 \log _{10} k$, we can get the following asymptotic formulas from (2.3), (2.4), and $(2.5)$.

$$
\text { For } K>20, \bar{R} \approx K+\frac{4.3429}{k^{2}}-2.5068
$$

TABLE 1 


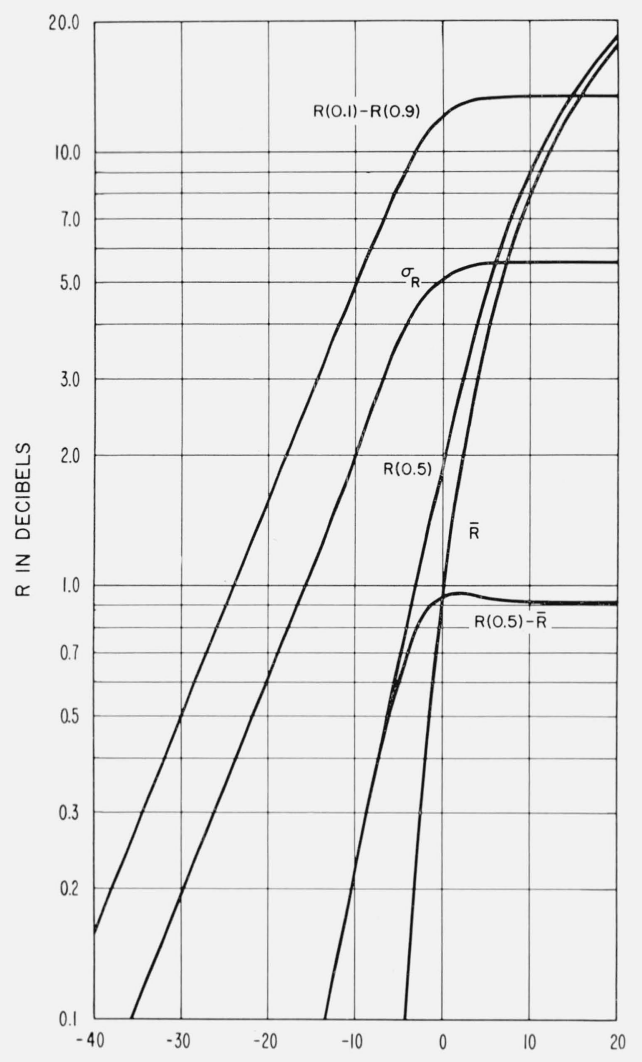

Figure 1. K in decibels.

and

$$
\begin{gathered}
\sigma_{R} \approx 4.3429 \sqrt{1.6449-1 /\left(2 k^{4}\right)} . \\
\text { For } K<-20, \bar{R} \approx 4.3429 k^{2} e^{-1 / k^{2}} \\
\sigma_{R} \approx 6.1418 k \sqrt{1+k^{2} / 2} .
\end{gathered}
$$

The magnitude of the error in these four expressions is less than $5(10)^{-4}$.

Values of the median, the mean, the difference between the mean and the median, and the standard deviation of $R$ are given in table 1 . These four quantities together with the interdecile range of $R$ are shown as functions of $K$ in figure 1. The median and interdecile range are taken from the paper by Norton et al. [1955].

\section{Appendix 1. Derivation of the Formulas for $\bar{R}$ and $\sigma_{R}$}

If we change to natural logarithms and make the substitution $r=k y$ in (2.1) and (2.2), we get

and

$$
\bar{R}=2\left(20 \log _{10} e\right) e^{-1 / k^{2}}[(\ln k) A(k)+B(k)],
$$

Here

$$
\overline{R^{2}}=2\left(20 \log _{10} e\right)^{2} e^{-1 / k^{2}}\left[(\ln k)^{2} A(k)+2(\ln k) B(k)+C(k)\right] .
$$

$$
\begin{gathered}
A(k)=\int_{0}^{\infty} y e^{-y^{2}} I_{0}(2 y / k) d y=\left[\int_{0}^{\infty} y^{2 a-1} e^{-y^{2}} I_{0}(2 y / k) d y\right]_{a=1}, \\
B(k)=\int_{0}^{\infty}(\ln y) y e^{-y^{2}} I_{0}(2 y / k) d y=\left[\frac{\partial}{\partial a} \frac{1}{2} \int_{0}^{\infty} y^{2 a-1} e^{-y^{2}} I_{0}(2 y / k) d y\right]_{a=1},
\end{gathered}
$$


and

$$
C(k)=\int_{0}^{\infty}(\ln y)^{2} y e^{-y^{2}} I_{0}(2 y / k) d y=\left[\frac{\partial^{2}}{\partial a^{2}} \frac{1}{4} \int_{0}^{\infty} y^{2 a-1} e^{-y^{2}} I_{0}(2 y / k) d y\right]_{a=1} .
$$

Using the Maclaurin series expansion for $I_{0}(2 y / k)$ and integrating term by term, we find that

$$
\int_{0}^{\infty} e^{-y^{2}} y^{2 a-1} I_{0}(2 y / k) d y=\frac{\Gamma(a)}{2}{ }_{1} F_{1}\left(a ; 1 ; 1 / k^{2}\right),
$$

where $\Gamma(x)$ is the gamma function and ${ }_{1} F_{1}(a ; c ; x)$ is a confluent hypergeometric function [Rainville, 1960].

In order to complete the evaluation of $A(k), B(k)$, and $C(k)$, we make use of the known series expansion for $-E i(-x)$ and of the series transformation

$$
e^{-x} \sum_{n=0}^{\infty} a_{n} \frac{x^{n}}{n !}=\sum_{n=0}^{\infty} b_{n} \frac{x^{n}}{n !}, \text { where } b_{n}=\Delta^{n} a_{0}
$$

is the $n$th forward difference of $a_{0}$; i.e., $\Delta^{0} a_{0}=a_{0}, \Delta^{1} a_{0}=a_{1}-a_{0}$, etc. Omitting any further details, we find that $\bar{R}$ is given by (2.3), and that

$$
\begin{aligned}
\overline{R^{2}}=\left(10 \log _{10} e\right)^{2}\{4 & (\ln k)^{2}+4(\ln k)\left[\ln \left(1 / k^{2}\right)-E i\left(-1 / k^{2}\right)\right]+\gamma^{2} \\
& \left.+\frac{\pi^{2}}{6}-2 \gamma\left[\gamma+\ln \left(1 / k^{2}\right)-E i\left(-1 / k^{2}\right)\right]+2 \sum_{n=1}^{\infty} \frac{(-1)^{n+1}\left(1 / k^{2}\right)^{n+1}}{(n+1) !(n+1)}\left(\sum_{j=1}^{n} \frac{1}{j}\right)\right\} .
\end{aligned}
$$

Combining (2.3) and (3.4), we get (2.4) for $\sigma_{R}$.

In order to prove (2.5), we derive a different expression for the series in (3.4). Observe that

where

$$
\sum_{n=1}^{\infty} \frac{(-1)^{n+1}(x)^{n+1}}{(n+1) !(n+1)}\left(\sum_{j=1}^{n} \frac{1}{j}\right)=\int_{1}^{x}\left[\sum_{n=1}^{\infty} \frac{(-1)^{n+1} t^{n}}{(n+1) !}\left(\sum_{j=1}^{n} \frac{1}{j}\right)\right] d t+C
$$

$$
C=\left[\sum_{n=1}^{\infty} \frac{(-1)^{n+1} x^{n+1}}{(n+1) !(n+1)}\left(\sum_{j=1}^{n} \frac{1}{j}\right)\right]_{x=1}=0.1827580 .
$$

Omitting any further details, except to note that use is made of the series expansions for $-E i(-x)$ and $E^{*}(x)$, and that we again use the series transformation (3.3), we find that

$$
\begin{aligned}
\sum_{n=1}^{\infty} \frac{(-1)^{n+1} x^{n+1}}{(n+1) !(n+1)}\left(\sum_{j=1}^{n} \frac{1}{j}\right)=-E i(-x)\left[E^{*}(x)-\gamma-\ln x\right]+E i(-1)\left[E^{*}(1)-\gamma\right] \\
+\int_{1}^{x} \frac{e^{t}}{t} E i(-t) d t-2 \int_{1}^{x} \frac{E i(-t)}{t} d t+\gamma \ln x+\frac{(\ln x)^{2}}{2}+C .
\end{aligned}
$$

Using this expression together with (2.3) and (3.4), we arrive at (2.5).

\section{Appendix 2. Evaluation of the Integrals $\int_{1}^{1 / k^{2}} \frac{-E i(-t)}{t} d t$ and}

$$
\int_{1}^{1 / k^{2}} \frac{e^{t}}{t}[-E i(-t)] d t
$$

The values of the integrals

$$
g\left(1 / k^{2}\right)=\int_{1}^{1 / k^{2}} \frac{E i(-t)}{t} d t \text { and } h\left(1 / k^{2}\right)=\int_{1}^{1 / k^{2}} \frac{e^{t}}{t}[-E i(-t)] d t
$$

given in table 2 for various values of $K$, were obtained as follows. The $i$ th entries of the table were computed in terms of the preceding entries by means of the formula

$$
\int_{1}^{x_{i}} f(t) d t=\left(\int_{1}^{x_{1}}+\int_{x_{1}}^{x_{2}}+\ldots+\int_{x_{i-1}}^{x_{i}}\right) f(t) d t
$$




\begin{tabular}{c|c|c|c}
\hline$K$ & $1 / k^{2}$ & $g\left(1 / k^{2}\right)$ & $h\left(1 / k^{2}\right)$ \\
\hline-0.96910013 & 1.2500000 & 0.0404747 & 0.123376 \\
-2 & 1.5848932 & .0679414 & .234919 \\
-4 & 2.5118864 & .0917103 & .402303 \\
-6 & 3.9810716 & .0971504 & .518481 \\
-6.9897000 & 5.0000000 & .0976706 & .561387 \\
-8 & 6.3095733 & .0978116 & .597259 \\
-9.0308999 & 8.0000000 & .0978393 & .626974 \\
-10 & 10.000000 & .0978428 & .649645 \\
-10.791812 & 12.000000 & .0978432 & .665007 \\
-12 & 15.848932 & .0978432 & .683943 \\
-14 & 25.118864 & .0978432 & .706139 \\
-16 & 39.810716 & .0978432 & .720383 \\
-18 & 63.095734 & .0978432 & .729470 \\
-20 & 100.00000 & .0978432 & .735245 \\
-30 & 316.22776 & .0978432 & .742039 \\
-35 & 1000.0000 & & \\
-40 & 3162.2776 & .0978432 & .744196 \\
$-\infty$ & 10000.000 & .0978432 & .744880 \\
& $\infty$ & .0978432 & .745096 \\
& & .0978432 & .745196 \\
\hline
\end{tabular}

where each of the integrals on the right-hand side of the equation was evaluated numerically using a 16-point Gaussian quadrature formula. Eight significant digits were used in the calculations and the results were rounded to six significant digits.

A different method for calculating the values of the integrals corresponding to $K=-40$ is presented here to check the values given in table 2. Due to the technique used in preparing the table, this will also be a check on the accuracy of the remaining tabular entries. First observe that

$$
\int_{1}^{10^{4}} \frac{e^{t}}{t}[-E i(-t)] d t=\int_{1}^{\infty} \frac{e^{t}}{t}[-E i(-t)] d t-0.0001+\epsilon
$$

where $|\epsilon|<0.5(10)^{-8}$, and

$$
\int_{1}^{10^{4}} \frac{-E i(-t)}{t} d t=\int_{1}^{\infty} \frac{-E i(-t)}{t} d t+\eta
$$

where $|\eta|<10^{-10}$. If we use the Maclaurin series for $e^{t}$, we get

$$
\int_{1}^{\infty} \frac{e^{t}}{t}[-E i(-t)] d t=\int_{1}^{\infty}-\frac{E i(-t)}{t} d t+\sum_{n=1}^{\infty} \frac{1}{n !} \int_{1}^{\infty} t^{n-1}[-E i(-t)] d t .
$$

From Erdélyi et al. [1953], we find that

$$
\int_{1}^{\infty} t^{n-1}[-E i(-t)] d t=\frac{\Gamma(n, 1)+E i(-1)}{n}=\frac{\Gamma(n)-\gamma(n, 1)+E i(-1)}{n}
$$

where $\Gamma(n, x)$ and $\gamma(n, x)$ are the incomplete gamma functions, and from Le Caine [1948] we get

Thus we find that

$$
\int_{1}^{\infty} \frac{-E i(-t)}{t} d t=\frac{\gamma^{2}+\frac{\pi^{2}}{6}}{2}-\sum_{n=0}^{\infty} \frac{(-1)^{n}}{n !(n+1)^{3}} .
$$

$$
\int_{1}^{10^{4}} \frac{-E i(-t)}{t} d t \approx 0.097843199
$$

with an error less than $0.5(10)^{-9}$ in magnitude. In the same way we may also show that

$$
\int_{1}^{12} \frac{-E i(-t)}{t} d t \approx 0.0978432^{-}
$$

with an error less than $0.5(10)^{-7}$ in magnitude. Omitting the details, we also get from the pre- 
ceding equations that

$$
\int_{1}^{10^{4}} \frac{e^{t}}{t}[-E i(-t)] d t \approx 0.097843199+\frac{\pi^{2}}{6}+E i(-1)\left[E^{*}(1)-\gamma\right]-\sum_{n=1}^{\infty} \frac{\gamma(n, 1)}{n ! n}-0.0001 \approx 0.74509596
$$

with an error less than $0.5(10)^{-8}$ in magnitude. It is seen that six significant digit accuracy is indicated for the values of the integrals given in table 2 .

Thanks are due to R. E. Wilkerson for checking the mathematics in this paper, and to Mrs. Gail J. Stifel for helping with the computations.

\section{References}

Erdélyi, A., W. Magnus, F. Oberhettinger, F. G. Tricomi, et al. (1953), Higher transcendental functions vol. 2 (McGraw-Hill Book Co. Inc., New York, N.Y.) (see eq (16), p. 144).

Le Caine, J. (1948), National Research Council of Canada, Division of Atomic Energy, Document No. MT-131 (NRC 1553) (see eq 1.6.1, p. 8).

Nakagami, Minoru (Oct. 1940), Study on the resultant amplitude of many vibrations whose phases and amplitudes are random, Nippon Elec. Comm. Eng. No. 22, 69-92 (see eq 118, p. 81).

Norton, K. A., L. E. Vogler, W. V. Mansfield, and P. J. Short (Oct. 1955), The probability distribution of the amplitude of a constant vector plus a Rayleigh-distributed vector, Proc. IRE 43, No. 10, 1354-1361.

Norton, K. A., E. L. Shultz, and H. Yarbrough (Jan. 1952), The probability distribution of the phase of the resultant vector sum of a constant vector plus a Rayleigh-distributed vector, J. Appl. Phys. 23, No. 1, $137-141$.

Rainville, E. D. (1960), Special functions (The Macmillan Company, New York, N. Y.) (see ex. 5, p. 128).

Rice, S. O. (July 1944), Mathematical analysis of random noise, Bell System Tech. J. 23, 282-332.

Rice, S. O. (Jan. 1945), Mathematical analysis of random noise, Bell System Tech. J. 24, 46-156. [Also published as Bell Telephone Monograph B 1589, and has been included in Selected papers on noise and stochastic processes, N. Wax, Ed. (Dover Publications, New York, N.Y., 1954) (see eq 3.10-11 in either the book edited by Wax, or Bell System Tech. J. 24.)]

U.S. National Bureau of Standards (1940), Tables of sine, cosine and exponential integrals, prepared by the Work Projects Administration for the Citv of New York, New York City, N.Y., volumes 1 and 2.

(Paper 68D4-358) 